\title{
Long-Term Clinical Outcomes of Transcervical Radiofrequency Ablation of Uterine Fibroids: The VITALITY Study
}

\author{
Jose Gerardo Garza-Leal, MD
}

\begin{abstract}
Objective: The aim of this research was to learn the long-term ( $>5$ years) clinical outcomes of transcervical radiofrequency ablation of uterine fibroids.

Materials and Methods: For this retrospective, single-arm, long-term data-collection study, 23 women with heavy menstrual bleeding secondary to fibroids were treated with transcervical radiofrequency ablation guided by integrated intrauterine sonography (using the Sonata ${ }^{\circledR}$ System, Gynesonics, Redwood City, CA). This study was within the 12-month Fibroid Ablation Study-EU clinical trial in Mexico. Symptoms were assessed using the Uterine Fibroid Symptom and Quality-of-Life's Symptom Severity Score (SSS) and Health-Related Quality of Life (HRQoL) subscales. Patients were queried regarding pregnancy and surgical reinterventions.

Results: Seventeen women (73.9\%) provided long-term follow-up information, with a mean of 64.4 months \pm 4.5 months (range: 57-73 months). From baseline, mean SSS decreased significantly from 64.9 16.9 to 27.6 \pm 36.1 , and mean HRQoL improved significantly from $27.2 \pm 22.4$ to $76.0 \pm 32.6(p=0.002$, and $p=0.0001$, respectively). There were no surgical reinterventions through the first 3.5 years post-treatment. There was an $11.8 \%$ incidence of surgical reinterventions over 5.4 years of average follow-up, with 2 hysterectomies occurring after 3.5 and 4 years postablation, respectively (event rate: $2.2 \%$ per year; $95 \%$ confidence interval; $0.3 \%, 7.9 \%$ ). Freedom from surgical reintervention at 1,2, and 3 years was $100 \%$, and, at 4 and 5 years, was $88.2 \% \pm 7.8 \%$. There was a single pregnancy occurring within the first year of treatment leading to a normal-term delivery by elective repeat cesarean section.

Conclusions: Transcervical radiofrequency ablation with the Sonata System produced substantial durable clinical benefits beyond 5 years with a low reintervention rate. (J GYNECOL SURG 35:19)
\end{abstract}

Keywords: radiofrequency ablation, intrauterine ultrasound, uterine fibroids

\section{Introduction}

$\mathbf{U}$ TERINE FIBROIDS are a common gynecologic disorder associated with heavy menstrual bleeding, pelvic pressure, urinary frequency, subfertility, and other symptoms, imposing a significant burden on women and their healthcare systems through the age of menopause. ${ }^{1,2}$ The most prevalent treatment for symptomatic fibroids remains hysterectomy, and fibroids are also the most frequent indication for benign hysterectomy. ${ }^{3}$ While hysterectomy is generally available, data from a national survey show that women are particularly resistant to invasive surgical options, often delaying treatment, on average, by 5 years, and that uterine-conserving procedures are of particular interest to women regardless of future desire for pregnancy. ${ }^{3}$ Access to less-radical treatment options, however, may not be sufficient in many regions, in part because of a lack of specialized surgical capabilities that limit treatment options, adequate payor coverage, and other factors. ${ }^{4}$

Transcervical radiofrequency ablation (RFA) with the Sonata ${ }^{\circledR}$ System (Gynesonics, Redwood City, CA; formerly

Department of Obstetrics and Gynecology, Hospital Universitario “Dr. José Eleuterio González" de Universidad Autonoma de Nuevo León, Monterrey, Mexico.

(C) Jose Gerardo Garza-Leal, 2018; Published by Mary Ann Liebert, Inc. This Open Access article is distributed under the terms of the Creative Commons Attribution Noncommercial License (http://creativecommons.org/licenses/by-nc/4.0/) which permits any noncommercial use, distribution, and reproduction in any medium, provided the original author(s) and the source are cited. 
known as the VizAblate ${ }^{\mathrm{TM}}$ System) is a minimally invasive, incisionless, uterine-conserving outpatient treatment for the majority of uterine fibroid types. ${ }^{5-8}$ Because of being guided by an integrated intrauterine sonography probe, this procedure allows the physician to visualize, target, and ablate all nonpedunculated fibroids. This includes deeper intramural, subserous, and hybrid fibroids (International Federation of Gynecology and Obstetrics [FIGO] type 3, type 4, type 5, type 6 , and type 2-5 [transmural]) that are not accessible via operative hysteroscopy, in addition to (nonpedunculated) submucous fibroids (FIGO type 1 and type 2 myomata). FIGO fibroid classification has been published previously. ${ }^{9}$ The Sonata System, which has a CE Mark, has been described in detail and provides a real-time graphical interface (SMART Guide ${ }^{\mathrm{TM}}$ ) that displays the location of each ablation on a live ultrasound image along with the extent of subablative thermal heating. 5,8

The Fibroid Ablation Study-EU (FAST-EU) was a 12month multicenter, prospective trial involving 7 clinical sites in the United Kingdom, The Netherlands, and Mexico. The trial was designed to establish effectiveness and confirm the safety of transcervical RFA of symptomatic uterine fibroids with the Sonata System. ${ }^{5,6}$ Fifty premenopausal women were enrolled and treated in the FAST-EU clinical trial and were followed through 12 months post-treatment. Patients were eligible if they had from 1-5 uterine fibroids of FIGO types $1,2,3,4$, and 2-5, between $1 \mathrm{~cm}$ and $5 \mathrm{~cm}$ in maximum diameter. All enrolled patients had to have at least 1 myoma that indented the endometrial cavity (e.g., type 1 , type 2 or type $2-5$ ), as these are most relevant to abnormal uterine bleeding (AUB).

The FAST-EU trial demonstrated significant reduction in menstrual bleeding along with diminished perfused and total fibroid volumes. There were also significant improvements in the Uterine Fibroid Symptom and Quality-of-Life (UFSQoL) subscales-the Symptom Severity Score (SSS) and Health-Related Quality of Life (HRQoL) - scores and a significant improvement in overall health status, as measured by the EuroQoL-5D (EQ-5D) questionnaire. These improvements were reported as early as 3 months postablation and were durable through 12 months. ${ }^{6}$ Treatment with the Sonata System was accompanied by low rates of serious adverse events and surgical reinterventions, with $92 \%$ of patients free from surgical reintervention at 12 months. To characterize the long-term clinical outcomes in patients treated with the Sonata System further, the VITALITY study was designed to collect retrospective followup information beyond 12 months of patients treated in the FAST-EU trial at an academic medical center in Monterrey, Mexico.

\section{Materials and Methods}

VITALITY was a retrospective, single-arm long-term $(>5$ years) data collection study conducted at a FAST-EU trial study site in Monterrey, Mexico.

The VITALITY study was designed to collect long-term clinical outcomes of patients with symptomatic fibroids who had been treated with transcervical RFA using the Sonata System. Patients who had been previously enrolled and treated with the Sonata System in the FAST-EU clinical trial were eligible for inclusion in the VITALITY study. The study protocol was approved by the ethics committee at the Hospital Universitario de Universidad Autonoma de Nuevo León, Monterrey, Mexico, and patients were contacted for participation. Informed consent was obtained from interested patients before enrollment in the study.

Patients who enrolled in the VITALITY study provided responses to the UFS-QoL questionnaire, which is used to assess both SSS and HRQoL, via these 2 subscales over the previous 3 months. Both subscales are normalized on a scale from 1 to 100 . Higher SSS scores are generally suggestive of a higher degree of symptom severity. ${ }^{10}$ The HRQoL subscale consists of 29 questions examining 6 dimensions (concern, activities, energy/mood, control, self-consciousness, and sexual function). Higher HRQoL scores are desirable as they reflect patients reporting a better HRQoL. The EQ-5D instrument was an additional questionnaire completed by patients in the FAST-EU clinical trial to obtain a descriptive profile of patient health status at baseline and post-procedure. The EQ-5D includes a visual analogue scale (VAS) from 0 to 100 , in which higher scores represent better health states, as well as 5 questions about mobility, self-care, usual activities, pain/discomfort, and anxiety/depression that are scored on a 1-5 scale (ranging from "no problem" to "extreme problems") and then converted to create a summary index that is representative of overall health status. ${ }^{11}$

Patients were also queried regarding surgical reinterventions for AUB as well as any pregnancy occurrences and outcome. While the original FAST-EU utilized Menstrual Pictogram (MP) bleeding diaries for patients to score their menstrual bleeding prospectively for the month, collection of the MP was not used for the VITALITY clinical trial to minimize the follow-up requirements for patients and increase the probability of successful long-term data collection. The UFS-QOL SSS subscale is used assess for the presence and significance of AUB. ${ }^{10}$

Statistical analyses were accomplished with SAS 9.3 (SAS, Cary, NC). Changes in variables were assessed using a paired $t$-test. Values were considered significant at the level of $\alpha=0.05$.

\section{Results}

Of the 23 patients treated in the FAST-EU trial at the site in Monterrey, Mexico, 17 (73.9\%) were able to be contacted and provided informed consent to be enrolled in the VITALITY study. The mean follow-up was 64.4 months \pm 4.5 months (range: 57-73 months). The initial characteristics of the patients and their fibroids are shown in Table 1.

At baseline, the mean SSS $(N=17)$ was $64.9 \pm 16.9$, and this significantly decreased to $27.6 \pm 36.1$ at the time of long-term follow-up $(p=0.002)$. The HRQoL, which had been $27.2 \pm 22.4$ on average at baseline $(N=17)$ significantly increased to $76.0 \pm 32.6$ at long-term follow-up $(p=0.0001)$. The HRQoL responses indicated significant improvements in all 6 dimensions (as noted above, these are concern, activities, energy/mood, control, self-consciousness, and sexual function) through the mean 64.4 months of follow-up.

Regarding the EQ-5D, there was significant mean improvement in the VAS scores from baseline to the long-term follow-up visit $(N=17)$. The baseline mean VAS score was $70.3 \pm 22.2$ and this significantly increased to $79.8 \pm 25.5$ $(p=0.042)$. The mean EQ-5D summary index increased 
Table 1. Initial Subjects' Characteristics

\begin{tabular}{lc}
\hline Characteristics & Statistics \\
\hline \# of subjects & 17 \\
Most frequent age range (in years) $^{\mathrm{a}}$ & $41-45^{\mathrm{a}}$ \\
Mean gravidity, parity $^{\mathrm{a}}$ & $3.4,2.9$ \\
Mean UFS-QoL SSS & $64.9 \pm 16.9$ \\
Mean UFS-QoL HRQoL & $27.2 \pm 22.4$ \\
Mean EQ-5D VAS & $70.3 \pm 22.2$ \\
Mean EQ-5D summary index & $0.79 \pm 0.23$ \\
Total \# of ablated fibroids & 35 \\
Mean \# of ablated fibroids per subject & 2.1 \\
Total \# of type 1 ablated fibroids & 7 \\
Total \# of type 2 ablated fibroids & 10 \\
Total \# of type 3 ablated fibroids & 2 \\
Total \# of type 4 ablated fibroids & 14 \\
Total \# of type 2-5 (transmural) ablated fibroids & 2 \\
Mean diameter of ablated fibroids (in cm) & $2.5 \pm 1.2$ \\
\hline
\end{tabular}

a'Subjects' ages were specified as a range by the site to protect subjects' privacy.

UFS-QoL, Uterine Fibroid Symptom and Quality-of-Life; HRQoL, Health-Related Quality of Life; EQ-5D, EuroQol-5D; VAS, visual analogue scale.

from $0.79 \pm .23$ at baseline to $0.83 \pm 0.26$ at long-term follow-up $(p=0.599)$.

No surgical reinterventions occurred through the first 3.5 years. At no time was any patient treated medically with selective progesterone-receptor modulators, gonadotropinreleasing hormone agonists, or any other drugs used to treat AUB. There were 2 hysterectomies reported, 1 after 3.5 years postablation and the other after $\sim 4$ years after treatment; both of these hysterectomies were performed to address AUB. The overall incidence for this 17-patient cohort was $11.8 \%$ over 5.4 years of average follow-up. This corresponds to an event rate of 2 reinterventions per 91 total patient-years of follow-up, or an event rate of $2.2 \%$ per year (95\% confidence interval: $0.3 \%, 7.9 \%$ ). Freedom from surgical reintervention at 1,2, and 3 years was $100 \%$ and, at 4 and 5 years, was $88.2 \% \pm 7.8 \%$ (Fig. 1).
Among the 17 patients, there was a single pregnancy, occurring within the first year of treatment, that was previously reported. ${ }^{12}$ This resulted in delivery by elective repeat cesarean section at term, with an unremarkable antenatal and perinatal course other than maternal gestational diabetes mellitus.

\section{Discussion}

Alternatives to hysterectomy, as they are not definitive treatments (unlike hysterectomy), are associated with the potential for fibroid recurrence due to further growth and/ or de novo growth of fibroids. When recurrent fibroids become symptomatic, surgical reintervention may be indicated. One study by Reed and colleagues found a $4.6 \%$ annual incidence of surgical reinterventions after myomectomy (including abdominal, laparoscopic, and hysteroscopic routes) during 2766.5 patient-years of follow-up. ${ }^{13}$ Among 628 eligible patients, there was a $23.5 \%$ cumulative incidence of surgical reintervention at 5 years, and this rose to $30 \%$ at 7 years. For laparoscopic myomectomy, specifically, Doridot and colleagues found a $4.6 \%$ surgical reintervention rate ( 8 of 173 patients) with a mean followup of 47.4 months. ${ }^{14}$ The cumulative recurrence risk was $12.7 \%$ at 2 years and $16.7 \%$ at 5 years. Differences in reintervention rates can be due to a variety of factors, including differences in length of follow-up, challenges in long-term surveillance, and treatment by general obstetrician/gynecologists versus specialists. ${ }^{13}$ Two randomized controlled trials of uterine artery embolization reported 5year reintervention rates of $28.4 \%-32.0 \%$. $^{15,16}$

The results of this single-center, retrospective datacollection study of transcervical RFA suggest its long-term ( $>5$ years) durability of reductions of symptoms and rate of surgical reintervention for fibroids could be at least comparable to other available alternatives to hysterectomy and might be greater. The FAST-EU clinical trial previously demonstrated significant reductions through 12 months with regard to heavy menstrual bleeding and other fibroid-associated symptoms along with marked improvement in HRQoL after

FIG. 1. Kaplan-Meier survival curve for surgical reintervention after treatment with the Sonata ${ }^{\circledR}$ System (Gynesonics, Redwood City, CA).

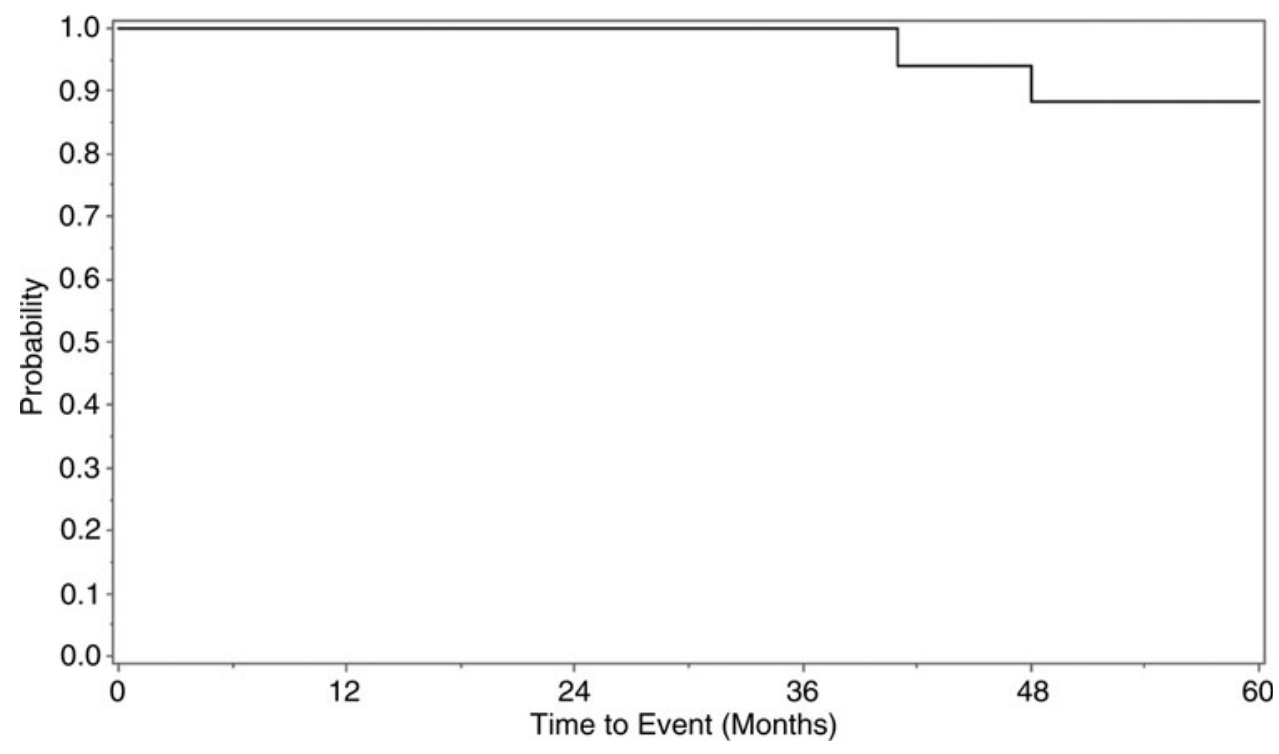


treatment with the Sonata System. ${ }^{6}$ The VITALITY study demonstrated that these significant improvements in SSS and HRQoL persisted through an average of 64.4 months after treatment with the Sonata System. Of note, no surgical reinterventions occurred within the first 3.5 years posttreatment, with an overall $2.2 \%$ event rate. This is consistent with other reports of low surgical reintervention rates among patients treated with a variety of single- and multiple-needle electrode RFA systems. ${ }^{17-20}$

As noted, long-term self-reported VAS scores for the EQ$5 \mathrm{D}$ questionnaire showed a significant improvement in health status, compared with baseline. The EQ-5D summary index, while not statistically significant, did indicate stable health status over 5 years of average follow-up, with a possible trend toward improvement. The results of the VAS and summary index were thus not discordant and were also consistent with the positive UFS-QoL results and reintervention rate.

The single pregnancy, which resulted in a positive perinatal outcome, was also encouraging. It should be noted that a desire for future fertility was an exclusion criterion in the FASTEU clinical trial. ${ }^{5}$ While a definitive risk profile regarding pregnancy after RFA in general remains to be established and compared against myomectomy, there is a growing body of evidence that supports the use of hyperthermic ablation for fibroids in women who desire fertility. ${ }^{12,21-24}$ The ability of the Sonata System to target and ablate uterine fibroids accurately in an incisionless fashion without resecting adjacent endometrium and myometrium holds promise as a fertilityconserving transcervical method of fibroid treatment.

Strengths of this study include a single operator, which provided consistency regarding perioperative management and long-term follow-up, as well as a high $(\sim 75 \%)$ patient long-term follow-up rate and inclusion of symptom and QoL data to complement the surgical reintervention histories. The study is limited by a relatively small patient cohort.

\section{Conclusions}

The VITALITY study demonstrated that transcervical RFA of uterine fibroids with the Sonata System can provide significant long-term (> 5-year) reduction of symptoms and improvement in QoL through more than 5 years, as well as a low rate of surgical reintervention $(0 \%$ reintervention rate through 3.5 years). These durable benefits are conferred through an incisionless, uterus-conserving outpatient procedure that can reduce the acute morbidity associated with more-invasive fibroid-treatment options.

\section{Acknowledgments}

David Toub, MD, MBA, contributed to the preparation of this article. Tami Crabtree, MS, provided a biostatistical analysis for this study.

\section{Author Disclosure Statement}

Dr. Garza's institution received research support from Gynesonics, Inc., for participation in both the FAST-EU and VITALITY clinical trials. Dr. Garza is a consultant to Gynesonics.

\section{References}

1. Fuldeore MJ, Soliman AM. Patient-reported prevalence and symptomatic burden of uterine fibroids among women in the United States: Findings from a cross-sectional survey analysis. Int J Womens Health 2017;9:403.

2. Zimmermann A, Bernuit D, Gerlinger C, Schaefers M, Geppert K. Prevalence, symptoms and management of uterine fibroids: An international internet-based survey of 21,746 women. BMC Womens Health 2012;12:6.

3. Borah BJ, Nicholson WK, Bradley L, Stewart EA. The impact of uterine leiomyomas: A national survey of affected women. Am J Obstet Gynecol 2013;209:319e1.

4. Al-Hendy A, Myers ER, Stewart E. Uterine fibroids: Burden and unmet medical need. Semin Reprod Med 2017;35:473.

5. Bongers M, Brolmann H, Gupta J, Garza-Leal JG, Toub D. Transcervical, intrauterine ultrasound-guided radiofrequency ablation of uterine fibroids with the VizAblate ${ }^{\circledR}$ System: Three- and six-month endpoint results from the FAST-EU study. Gynecol Surg 2015;12:61.

6. Brolmann H, Bongers M, Garza-Leal JG, et al. The FASTEU trial: 12-month clinical outcomes of women after intrauterine sonography-guided transcervical radiofrequency ablation of uterine fibroids. Gynecol Surg 2016;13:27.

7. Jones S, O'Donovan P, Toub D. Radiofrequency ablation for treatment of symptomatic uterine fibroids. Obstet Gynecol Int 2012;2012:194839.

8. Toub DB. A new paradigm for uterine fibroid treatment: Transcervical, intrauterine sonography-guided radiofrequency ablation of uterine fibroids with the Sonata System. Curr Obstet Gynecol Rep 2017;6:67.

9. Munro MG, Critchley HO, Broder MS, Fraser IS; FIGO Working Group on Menstrual Disorders. FIGO classification system (PALM-COEIN) for causes of abnormal uterine bleeding in nongravid women of reproductive age. Int $\mathbf{J}$ Gynaecol Obstet 2011;113:3.

10. Spies JB, Coyne K, Guaou Guaou N, Boyle D, SkyrnarzMurphy K, Gonzalves SM. The UFS-QOL, a new diseasespecific symptom and health-related quality of life questionnaire for leiomyomata. Obstet Gynecol 2002;99: 290.

11. The EuroQol Group. EuroQol—a new facility for the measurement of health-related quality of life. Health Policy 1990;16:199.

12. Garza-Leal JG, León IH, Toub D. Pregnancy after transcervical radiofrequency ablation guided by intrauterine sonography: Case report. Gynecol Surg 2014;11:145.

13. Reed SD, Newton KM, Thompson LB, McCrummen BA, Warolin AK. The incidence of repeat uterine surgery following myomectomy. J Womens Health (Larchmt) 2006; 15:1046.

14. Doridot V, Dubuisson JB, Chapron C, Fauconnier A, Babaki-Fard K. Recurrence of leiomyomata after laparoscopic myomectomy. J Am Assoc Gynecol Laparosc 2001; $8: 495$.

15. van der Kooij SM, Hehenkamp WJ, Volkers NA, Birnie E, Ankum WM, Reekers JA. Uterine artery embolization vs hysterectomy in the treatment of symptomatic uterine fibroids: 5-year outcome from the randomized EMMY trial. Am J Obstet Gynecol 2010;203:105e1.

16. Moss JG, Cooper KG, Khaund A, et al. Randomised comparison of uterine artery embolisation (UAE) with surgical treatment in patients with symptomatic uterine fibroids (REST trial): 5-year results. BJOG 2011;118:936. 
17. Ghezzi F, Cromi A, Bergamini V, Scarperi S, Bolis P, Franchi M. Midterm outcome of radiofrequency thermal ablation for symptomatic uterine myomas. Surg Endosc 2007;21:2081.

18. Iversen H, Dueholm M. Radiofrequency thermal ablation for uterine fibroids: Long-term clinical outcomes and reinterventions. J Minim Invasive Gynecol 2017;24:1020.

19. Cho HH, Kim JH, Kim MR. Transvaginal radiofrequency thermal ablation: A day-care approach to symptomatic uterine myomas. Aust N Z J Obstet Gynaecol 2008;48:296.

20. Berman JM, Guido RS, Garza-Leal JG, et al. Three-year outcome of the HALT trial: A prospective analysis of radiofrequency volumetric thermal ablation of myomas. $\mathrm{J}$ Minim Invasive Gynecol 2014;21:767.

21. Keltz J, Levie M, Chudnoff S. Pregnancy outcomes after direct uterine myoma thermal ablation: Review of the literature. J Minim Invasive Gynecol 2017;24:538.

22. Rabinovici J, David M, Fukunishi H, et al. Pregnancy outcome after magnetic resonance-guided focused ultrasound surgery (MRgFUS) for conservative treatment of uterine fibroids. Fertil Steril 2010;93:199.
23. Berman JM, Bolnick JM, Pemueller RR, Garza-Leal JG. Reproductive outcomes in women following radiofrequency volumetric thermal ablation of symptomatic fibroids: A retrospective case series analysis. J Reprod Med 2015;60(5-6):194.

24. Berman JM, Puscheck EE, Diamond MP. Full-term vaginal live birth after laparoscopic radiofrequency ablation of a large, symptomatic intramural fibroid: A case report. J Reprod Med 2012;57(3-4):159.

Address correspondence to: Jose Gerardo Garza-Leal, MD Department of Obstetrics and Gynecology Hospital Universitario "Dr. José Eleuterio González," Av. Francisco I. Madero y Dr. Eduardo Aguirre Pequeño Col. Mitras Centro Monterrey Nuevo León 64460

México

E-mail: drjoseggarza@prodigy.net.mx 